This method of examining the retinal vascular system would appear to open new possibilities in the investigation of various fundal states including the relationship between the colour of the optic disc and its capillary content, the state of the capillary bed especially at the edge of the disc in glaucoma, and its relationship to visual field defects, new-vessel formation in the retina and vitreous, the extent of the capillary free zone at the macula in various conditions, etc.

It also affords an opportunity of investigating the degree of collateral circulation existing in the optic nerve head and choroid.

\title{
Summary
}

1. A method is described of injecting the retinal vascular system in the freshly enucleated eye.

2. Indication is given of the possible usefulness of this new technique.

\section{SEVERE LESION OF THE VISUAL PATH IN PREGNANCY*}

BY

\author{
G. PERÉMY
}

BUDAPEST

RECENTLY I had the opportunity to review the condition of a patient whom I saw for the first time nearly 20 years ago. The case seems to be a very rare one. The case described by Lawford Knaggs more than half a century ago is closely similar to the one I am going to relate, but in the literature of the last years, as far as I had access to it, I could not trace another of that kind.

Case history.-The patient was sent in 1930 by the ophthalmologist to the First Medical Clinic of the University of Budapest for consultation on account of the rapid deterioration of the vision of her left eye. She was at that time 40 years of age and in the fourth month of her sixth pregnancy. She had already gone through three pregnancies when she accidentally became aware of the complete loss of the vision of her right eye. This happened six years earlier. In her earlier history there was nothing which could have been brought into relationship with her condition at that time, except for an apparently insignificant, slight headache which she had sometimes since her 'teens. The medical examination revealed a normal physical condition, including blood-pressure, urine and blood picture. The Wassermann test was negative. The ophthalmological data were as follows: The right pupil reacted to light only consensually, the left one only directly. Amaurosis and primary optic atrophy were found in the right eye. With the left eye she could count fingers at seven feet.' The margin of the left optic disc was sharp,

* Received for publication, August 28, 1948. 
and it was somewhat reddish, no papilloedema was to be observed. There was a complete central scotoma for all colours, the visual field was contracted in a high degree for red, less for white. The patient had a marked squint as a result of the amaurosis. Examination of the nervous system revealed no abnormalities. The senses of smell and taste were also normal. The pressure and the composition of the cexebrospinal fluid were normal. X-ray plates of the skull showed no signs of increased intracranial pressure. The size and the shape of the sella turcica were normal. The sinuses were clear.

The optic atrophy on the one side and the retrobulbar neuritis on the other were judged to be caused by the pregnancies. The interruption of the pregnancy of the time seemed inevitable, yet the patient left the clinic and induction was not performed until one month later, after further deterioration of her vision. I asked her to come to the clinic for re-examination. She did so two and a half years later. She said she had experienced an improvement of her vision for some months after the interruption of her pregnancy, and since then it was unchanged. She had still her menstrual flow regularly. She had no complaints. A complete loss of the temporal half of the left visual field was found. The perception of red and green in the centre of the nasal half was retained. On being asked, she related that for some time she was forced to turn her head excessively to the left when crossing a street, or else she was unaware of vehicles coming from that side. An X-ray plate of the sella showed it to be unaltered. She had put on some weight, but there were no signs of abnormalities of nervous or hypophyseal origin. The urine and the blood-pressure were normal. The findings of the ophthalmologist were as follows: The condition of the right eye was the same as at the examination two and a half years earlier. The vision of the left eye was 5/10. The left optic disc was pale. There was a temporal hemianopia. The nasal colour-fields were normal.

On re-examining the patient some weeks ago, I found that her ophthalmological condition remained unchanged. In particular the nasal colour-fields were intact. Neither could I find any other change in the condition of the patient.

There could be no doubt that this severe lesion of the visual path had been brought about by pregnancies. It could have been caused by toxic damage to the optic fibres, or by pregnancy-enlargement of the adenohypophysis, though both possibilities were equally hypothetical. The later ophthalmological findings revealed a damage to the optic chiasma or to the right optic tract too (the right eye already being amaurotic). On the basis of this condition I suggested that there might have been some benign cystic growth of the hypophysis which exerted a pressure on the chiasma mediated through the pregnancyenlargement of the adenohypophysis. But the course of the disease and the present condition of the patient make such a hypothesis extremely unlikely. Certainly signs of increased intracranial pressure were never observed, and no change in the size and shape of the sella occurred. Neither did any other nervous or endocrine disorder develop. In a case of such a strong and repeatedly exerted pressure on the visual path which resulted in the atrophy of one optic nerve and one optic tract, other signs too should be expected, e.g., a disturbance of smell, and the cardinal signs of hypophyseal-diencephalic hypofunction. Yet no sign of that kind appeared in more than 20 years. Thus we are led to assume repeated toxic damage to the optic fibres resulting from pregnancy, which first brought about a complete atrophy of one optic nerve, and later 
affected the remaining fibres of one optic tract too. Toxic damage to the optic fibres including that which is associated with pregnancy is exerted in most cases on the retrobulbar part of the axial fibres of the optic nerve, and thus arises what is known as retrobulbar neuritis. The damage to the optic fibres originating in the left retina of the patient was initiated in this way. This may support the assumption of a toxic origin, though the impairment of central vision may also be an early sign of pressure. The peculiar feature of the case presented here is that the toxic damage should extend so far along the visual path. The multiplicity of pregnancies may account for this. In such cases the task of differentiating a toxic state from that caused by a tumour in or near the sella may arise. As I tried to show, the hemianopic defect of the visual field does not prove beyond doubt that it is caused by the pressure of a neoplasm; it may be the result of the same toxic damage to the optic tract as that which affected the optic nerve. Cases may occur in which the alternative-pressure or toxic damage-cannot be decided for a while. Decision may sometimes be possible only in the later course of the disease. Fortunately in cases of pregnancy such uncertainty does not imply any therapeutical dilemma, since termination of the pregnancy is warranted in the case of an alleged tumour especially if the impairment of vision seems severe; and it is known that the growth of a hypophyseal adenoma may be stimulated by simultaneous pregnancy.

REFERENCE

KNAGGS, LAWFORD (1896).-Brit. Med. J., 330.

\title{
AN UNUSUAL CONGENITAL DEFECT*
}

BY

\author{
AllaN H. BRIgGs and D. W. MCLEAN
}

LINCOLN

FOLKESTONE

DAPhNe S. was brought to one of us (A.H.B.) at the age of fourteen days, as the parents had noticed an abnormality of the right eye since birth. The child was normal and healthy in all other respects, and there was no family history of known ophthalmic defect. The pregnancy and labour were apparently normal.

On examination, difficult in so small an infant, it was found that the lids and adnexa were normal. The eye was white and of

* Received for publication, November 16, 1948. 